\title{
Clinical significance of proliferative potential of occult metastatic cells in bone marrow of patients with breast cancer
}

\author{
J-Y Pierga*, , C Bonneton ${ }^{2}$, H Magdelénat ${ }^{3}$, A Vincent-Salomon ${ }^{4}$, C Nos $^{5}$, P Pouillart' and J-P Thiery ${ }^{2}$ \\ 'Medical Oncology Department, Institut Curie, 75248 Paris Cedex 05, France; '2UMR I44 CNRS, Institut Curie, 75248 Paris Cedex 05, France; \\ ${ }^{3}$ Translational Research Laboratory, Institut Curie, 75248 Paris Cedex 05, France; ${ }^{4}$ Pathology Department, Institut Curie, 75248 Paris Cedex 05, France; \\ ${ }^{5}$ Surgery Department, Institut Curie, 75248 Paris Cedex 05, France
}

\begin{abstract}
There is increasing statistical evidence that the presence of tumour cells in bone marrow detected by immunocytochemistry represents an important prognostic indicator in breast cancer, but their individual capacity to become clinical metastases is unknown. The aim of this study was to assess the proliferative capacity of these occult metastatic cells in the bone marrow of patients with various stages of breast cancer. We obtained bone marrow aspirates from 60 patients with breast cancer before treatment with chemotherapy: 17 stage II, I 2 stage III and 3 I stage IV. After bone marrow culture for 6-34 days (median: I 7 days) under specific cell culture conditions, viable epithelial cells were detected by cytokeratin staining in 40 patients (66\%). Expansion of tumour cells was poorly correlated with tumour cell detection on primary screening $(P=0.06)$. There was a nonsignificant correlation between the number and the presence of expanded tumour cells and the UICC stage of the patients. On primary screening, tumour cell detection was positive in $56 \%$ of patients and was correlated with clinical UICC stage $(P=0.0$ I). However, with a median follow-up of 23 months, expansion of tumour cells from bone marrow was associated with decreased patient survival $(P=0.04)$, whereas the survival difference according to detection of CK-positive cells on primary screening was not statistically significant. In conclusion, viable tumour cells can be detected in the bone marrow of breast cancer patients. Their proliferative potential could be predictive of outcome and deserves further investigation.
\end{abstract}

British Journal of Cancer (2003) 89, 539-545. doi: I 0.1038/sj.bjc.660 I I2I www.bjcancer.com

(c) 2003 Cancer Research UK

Keywords: breast cancer; bone marrow; cytokeratin; culture

The clinical importance of occult tumour cells in the bone marrow of breast cancer patients has been demonstrated in several prospective studies, and represents an independent prognostic factor for distant relapse and overall survival (Harbeck et al, 1994; Diel et al, 1996; Mansi et al, 1999; Braun et al, 2000b; Gebauer et al, 2001; Gerber et al, 2001). Immunocytochemical (ICC) detection of epithelial cells in the bone marrow of breast cancer patients has been performed with a variety of antibodies, but the specificity and clinical relevance of the markers used to characterise epithelial cells remain controversial (Braun et al, 1998; Funke and Schraut, 1998). However, the simple detection of CK-positive cells does not provide any information about the proliferative potential of disseminated cancer cells. No marker is available to differentiate between cells that will die, those in a quiescent state and those with metastatic and proliferative potentials. Very little is known about the biological features of these cells. Currently available data suggest that CK-positive cells in bone marrow aspirates of cancer patients represent a selected, but nevertheless heterogeneous population of dormant (G0-phase) cancer cells (Braun and Pantel, 1999). Only a small fraction of disseminated tumour cells in bone marrow expressed a proliferative marker (Ki-67 or p120) in

\footnotetext{
*Correspondence: Dr J-Y Pierga; Département d'Oncologie Médicale, Institut Curie, 26 rue d'Ulm, 7523I Paris Cedex 05, France;

E-mail: jean-yves.pierga@curie.net

Received 10 January 2003; revised 28 April 2003; accepted 7 May 2003
}

double-staining studies (Pantel et al, 1993). The dormant state of these cells may be one explanation for the relative resistance of micrometastatic tumour cells to chemotherapy (Braun et al, 2000a).

In a recent study, Solakoglu et al (2002) cultured bone marrow samples from 153 patients with various types of carcinoma (breast, prostate, colon and kidney). Viable epithelial cells were detected by cytokeratin staining in $81 \%$ of patients with no known distant metastases. Marked expansion of tumour cells was also correlated with decreased patient survival. Extensive cell culture experiments have also shown that cells disseminating into bone marrow have a time-limited proliferative potential (Pantel et al, 1995).

The present study is an attempt to investigate the clinical relevance of the in vitro proliferative potential of CK-positive tumour cells by culturing under specific tissue culture conditions and to compare the detection of these expended cells to 'standard' ICC detection in bone marrow using anticytokeratin antibodies.

\section{PATIENTS AND METHODS}

\section{Patients}

Sixty consecutive patients with primary or metastatic breast cancer were included in the study between 1999 and 2001 after giving their written informed consent. All patients were treated at the Institut Curie and follow-up data were obtained prospectively. The 
clinicopathological data and information concerning treatment modalities of the patients are summarised in Table 1. Their median age was 52 years (range: $36-74$ years). Histology showed 51 ductal, eight lobular, one apocrine and one undifferentiated carcinomas. Forty-one tumours were hormone receptor positive, 15 were negative and four were undetermined. UICC stages were 17 stage II, 12 stage III and 31 stage IV. TUICC classification of tumour sizes for nonmetastatic patients was three $\mathrm{T} 1,12 \mathrm{~T} 2$, two $\mathrm{T} 3$, four $\mathrm{T} 4$ not d, and six inflammatory breast cancers (T4d). Bone marrow sampling was performed in 14 patients before neoadjuvant chemotherapy, in 12 before adjuvant chemotherapy, based on four to six cycles of docetaxel-doxorubicin or epirubicin, cyclophosphamide \pm 5 FU. Only three nonmetastatic patients did not receive adjuvant chemotherapy. The median follow-up was 23 months (range: 13-31 months) after sampling. Bone marrow aspirates $(4-5 \mathrm{ml}$ per sample) were obtained from both anterior iliac crests preoperatively in five patients or before starting chemotherapy (neoadjuvant or for metastatic disease) in 55 patients. One bone marrow aspirate was obtained under local anaesthesia from the posterior iliac crest (39 patients including all the stage IV patients) or by sternal tap ( $4-5 \mathrm{ml}$ per sample) (16 patients).

\section{Bone marrow preparation}

Three to five millilitres of bone marrow aspirate were collected and stored on EDTA (Vacutainer). Components of the bone marrow aspirate were processed under laminar flow. Each sample was diluted in Hanks (Gibco Brl UK) and separated by Ficoll/Hypaque density centrifugation (Sigma; density, $1.077 \mathrm{~g} \mathrm{ml}^{-1}$ ) in Leucosep tubes (Polylabo) $\left(830 \mathrm{~g}, 15 \mathrm{~min}, 20^{\circ} \mathrm{C}\right)$. The mononuclear cell (MNC) layer was harvested from each tube, combined, diluted in Hanks and centrifuged at $360 \mathrm{~g}, 5 \mathrm{~min}$ at $20^{\circ} \mathrm{C}$ in a $50 \mathrm{ml}$ conical tube. The cells were resuspended in PBS solution $+0.1 \%$ BSA (bovine serum albumin). Cell counts were performed on aliquots diluted in $3 \%$ acetic acid for red cell lysis. An aliquot of aspirate was tested for the presence of CK-positive tumour cells (primary screening). The remaining bone marrow cells were cultured and then rescreened for CK expression (culture screening).

\section{Primary screening}

Mononuclear cells were resuspended at $1 \times 10^{6} \mathrm{ml}^{-1}$. One millilitre of the cell suspension (approximately $10^{6}$ cells) was cytocentrifuged onto polylysinated slides at $580 \mathrm{~g}$ twice for $3 \mathrm{~min}$ (Hettich Universal 16A cytocentrifuge) (Schwartz, 1991). The supernatant was carefully removed from each slide after the first cytocentrifugation and the slides were allowed to dry in air overnight. Slides were stored at room temperature before staining or were stored at $-20^{\circ} \mathrm{C}$ and then at $-80^{\circ} \mathrm{C}$ until staining.

\section{Cell culture and culture screening}

Bone marrow cells were cultured as described by Pantel et al (1995) and Solakoglu et al (2002). MNCs $\left(1 \times 10^{7}\right.$ to $\left.3 \times 10^{7}\right)$ were initially plated in culture flasks coated with an extracellular matrix (Paesel \& Lorei, Frankfurt, Germany). The culture medium contained RPMI 1640 supplemented with $10 \%$ FCS, $10 \mu \mathrm{g} \mathrm{ml}^{-1}$ transferrin, $5 \mu \mathrm{g} \mathrm{ml}^{-1}$ insulin, $2 \mathrm{mM}$ glutamine, $10 \mathrm{ng} \mathrm{ml}^{-1}$ basic Fibroblastic Growth Factor (Boehringer Mannheim, Germany) and $10 \mathrm{ng} \mathrm{ml}^{-1}$ recombinant human epidermal growth factor (Boehringer Mannheim, Germany). The cells were cultured under 5\% $\mathrm{CO}_{2}$ and reduced oxygen (4\%). Before confluence, the adherent cells (including the epithelial tumour cells) were removed by trypsination. To determine the number of CK-positive cells, an aliquot containing 1 to $30 \times 10^{5}$ cells was centrifuged onto glass slides and immunocytochemically stained (culture screening) using the method described above. In some cases, cells were

Table I Patient characteristics

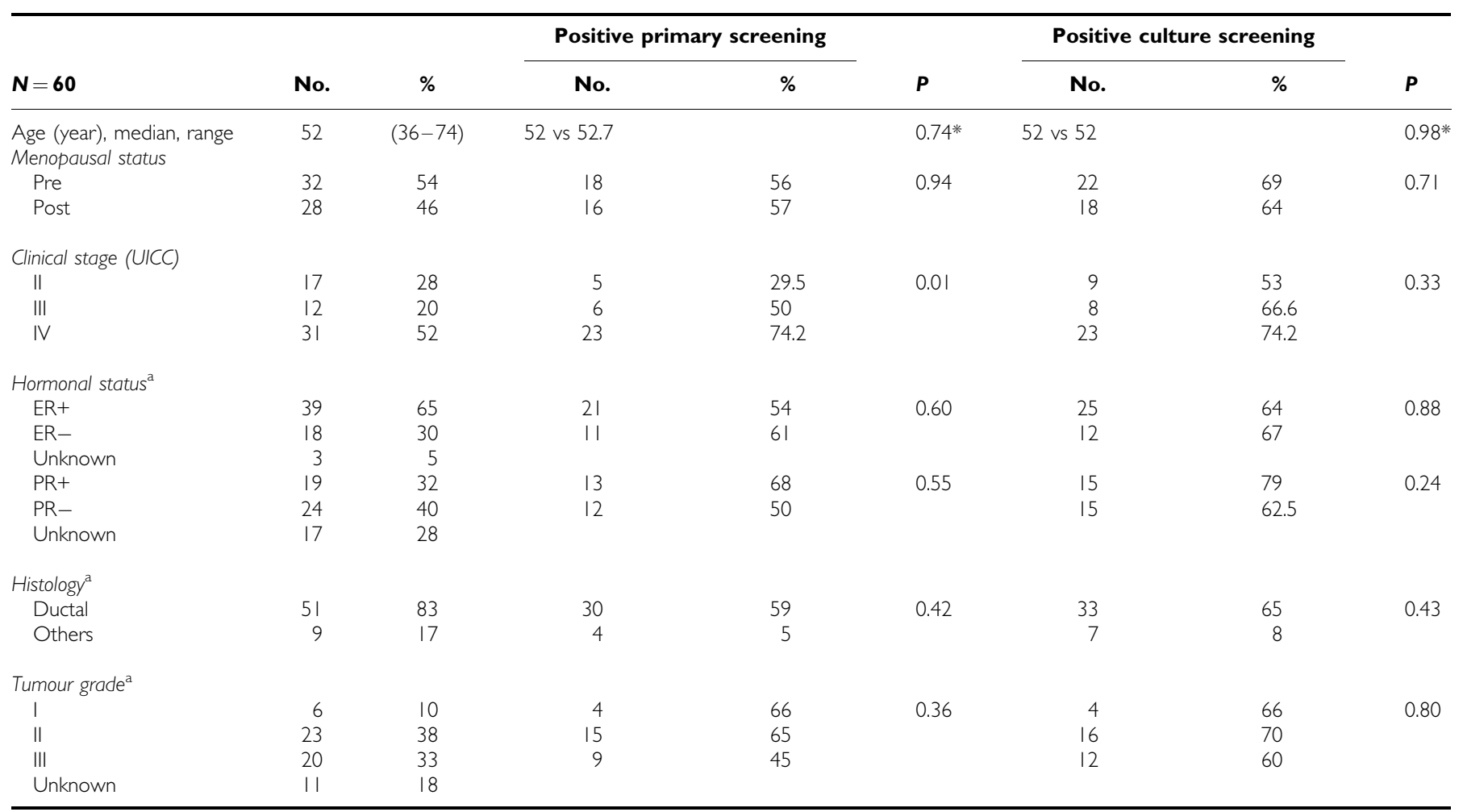

*Student's t-test. ${ }^{a}$ At initial diagnosis. 
transferred into new flasks. Two to three slides were stained with A45 B/B3 and one to two slides were used as controls with irrelevant IgG1. The number of in vitro expanded CK-positive cells was calculated: number of $\mathrm{CK}$ cells detected on slides after trypsination on culture screening according to the total number of MNCs per slide (from $10^{4}$ to $10^{5}$ ) divided by the total number of MNCs cultured at the time of sample collection. Results are expressed as the number of CK cells $/ 10^{6}$ MNCs.

\section{Immunocytochemical staining}

The pancytokeratin (CK) monoclonal antibody A45-B/B3 (Micromet, Germany and Chromavision, USA), which recognises several cytokeratin epitopes CK 8, CK 18 and CK 19, was applied for epithelial cell detection (Stigbrand et al, 1998). The immunostaining procedure was standardised by using a Cadenza (Shandon) automat. Before staining, cytospots were fixed with $4 \%$ paraformaldehyde for $5 \mathrm{~min}$ then dried for $15 \mathrm{~min}$ at room temperature. Endogenous alkaline phosphatase was then blocked with TBS solution with $2 \% \mathrm{AB}$ serum (15 min) (Sanofi Diagnostics Pasteur, USA) and $2 \%$ levamisole. This solution was used to dilute primary and secondary antibodies. After blocking, the slides were incubated with the primary antibody A45 B/B3 for $40 \mathrm{~min}$ $\left(2 \mu \mathrm{g} \mathrm{ml}^{-1}\right)$. Control slides were incubated under the same conditions with a mouse monoclonal anti-FITC IgG1 (one out of 1250) (Sigma Immuno Chemicals, USA). Slides were incubated for $20 \mathrm{~min}$ with secondary polyclonal rabbit anti-mouse antibody (Dako, USA). After each step, the slides were rinsed for $5 \mathrm{~min}$ in TBS $1 \times$ solution. Immune complexes were revealed by the alkaline phosphatase-anti-alkaline phosphatase technique (Dako, USA) (one out of 50) for $25 \mathrm{~min}$ (Cordell et al, 1984). The chromogenic reaction was performed for $20 \mathrm{~min}$ with a colorimetric substrate of Fuchsin solution $(2.5 \%$ in $2 \mathrm{~N} \mathrm{HCl})(\mathrm{New}$ Fuchsin, Sigma) with $4 \% \mathrm{NaNO}_{2}, 8 \% \beta$-naphthol (Sigma, USA) and $2 \%$ levamisole (Dako, USA). Cells were counterstained with Mayer's haematoxylin (1 min) (Sigma, USA) diluted to $1: 3$ in distilled water. The specimen was then rinsed under running water for $5 \mathrm{~min}$ and then in TBS. Slides were coverslipped using Faramount mounting medium (Dako, USA). Mononuclear cells $\left(3 \times 10^{6}\right)$ were evaluated for each patient and for each bone marrow sample. Negative controls, stained with anti-FITC monoclonal mouse antibody, were performed on an equivalent number of cells (i.e. three slides, $3 \times 10^{6} \mathrm{MNCs}$ ) for each patient.

Positive controls were obtained with bone marrow from normal donors undergoing orthopaedic surgery (Cochin Hospital), spiked with SKBR3 or MCF7 cell lines, $10-10^{2}$ for $10^{6}$ MNCs per cytospot. One positive control slide and one negative control slide were added to each series of 20 stained slides in the automated device.

\section{CK-positive cell detection by digital microscopy}

The ACIS (ChromaVision Medical Systems, Inc.). is a computerised microscope, which includes an image processing system that has been optimised for the detection of rare carcinoma cells in specimens (Bauer et al, 2000). The application software supplied with the instrument starts by scanning a microscope slide at low magnification $(\times 10)$. The instrument then returns to objects originally identified by their stain for a second analysis at higher magnification $(\times 40$ or $\times 60)$. In this case, more sophisticated image analysis of colour and morphometric characteristics is performed in order to exclude cellular debris, large clumps and cells with morphological features typical of normal haematological MNCs as opposed to CK-positive carcinoma cells. Cellular objects that meet colour- and morphometry-based criteria for probable tumour cells are collected and presented as montage images for review and classification by a pathologist or another investigator (JYP). Criteria for evaluation of immunostained cells in bone marrow were adapted from Borgen et al (1999) based on the results of the European ISHAGE Working Group for standardisation of tumour cell detection.

\section{Statistical methods}

Patient characteristics were prospectively recorded on the Institut Curie medical files. Differences between treatment groups were analysed by $\chi^{2}$ tests for categorical variables and $t$-tests for continuous variables, and the Kruskal-Wallis test was used for nonparametric comparisons. Survival time and disease-free survival time were measured from the date of bone marrow aspiration until the date of death or last follow-up. Survival curves were determined using a Kaplan-Meier product-limit method (Kaplan and Meier, 1958). Statistical significance between groups was assessed using the log-rank test. Statistical analyses were performed by Statview software (SAS Institute Inc., 1998).

\section{RESULTS}

\section{Expansion of bone marrow cells}

From August 1999 to March 2001, 60 bone marrow samples were successfully cultured. Cultures were stopped between 6 and 34 days (median: 17 days). An example of positive results is given in Figure 1 and Figure 2. No correlation was observed between the number of CK-positive cells detected in the bone marrow sample (primary screening) and the number of CK-positive cells in the culture screening $(P=0.19)$. Thirty-four $(56 \%)$ patients presented a positive primary screening result, while $40(66 \%)$ of these patients presented a positive culture screening result. CK-positive cell detection on primary screening was not strongly correlated with detection on culture screening $(P=0.06)$. Fourteen cases $(23 \%)$ initially negative on primary screening became positive on culture screening. Inversely, eight cases classified as positive on the initial screening, failed to produce any CK-positive cells $(13 \%)$ on culture screening (Table 2).

\section{Correlation with tumour stage}

The correlation between primary screening and tumour stage was statistically significant. Among the 34 patients with detectable CKpositive cells on primary screening, five of $17(29 \%)$ had stage II tumours, six of $12(50 \%)$ had stage III tumours and 23 of $31(74 \%)$ had stage IV tumours, according to the UICC classification (International Union Against Cancer) $\left(\chi^{2}\right.$ test, $\left.P=0.01\right)$. As shown in Table 3, the number of CK-positive cells on initial screening was correlated with UICC stage $(P=0.0013)$. No significant correlation was observed between the results of culture screening and tumour stage $(P=0.21) \quad$ (Table 3$)$. Tumour cell expansion was not correlated with hormonal receptor status $(P=0.88)$ or histology (ductal $v s$ lobular) $(P=0.43)$ or tumour grade $(P=0.80)$ (Table 1$)$. Interestingly, not all cell cultures of the 31 patients with clinically overt metastases harboured CK-positive cells: eight cases were negative for culture (26\%) (Table 2). Cultures were also negative in four cases of clinical metastatic disease with positive initial screening (see Table 2).

\section{Correlation with clinical outcome}

The median follow-up was 23 months. Thirteen deaths have occurred, all in metastatic patients. The median survival has not been reached in view of this short median follow-up. Statistical analysis (log-rank test) showed that primary detection of CKpositive cells in 34 patients was not significantly correlated with an increased risk of cancer-related death $(P=0.15)$ (Figure 3B). Forty patients had positive culture screening and 12 of them have died. Two of these patients had a negative primary screening. The difference in overall survival between the CK-positive group and 

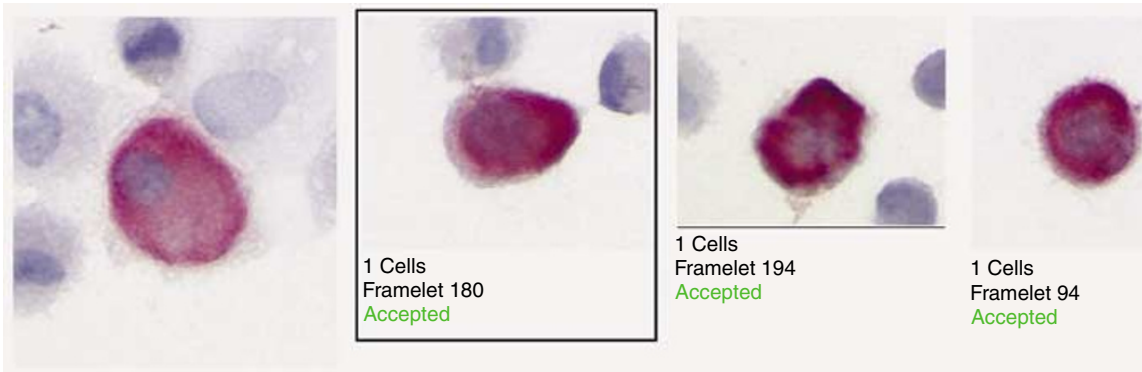

1 Cells
Framelet 167
Accepted
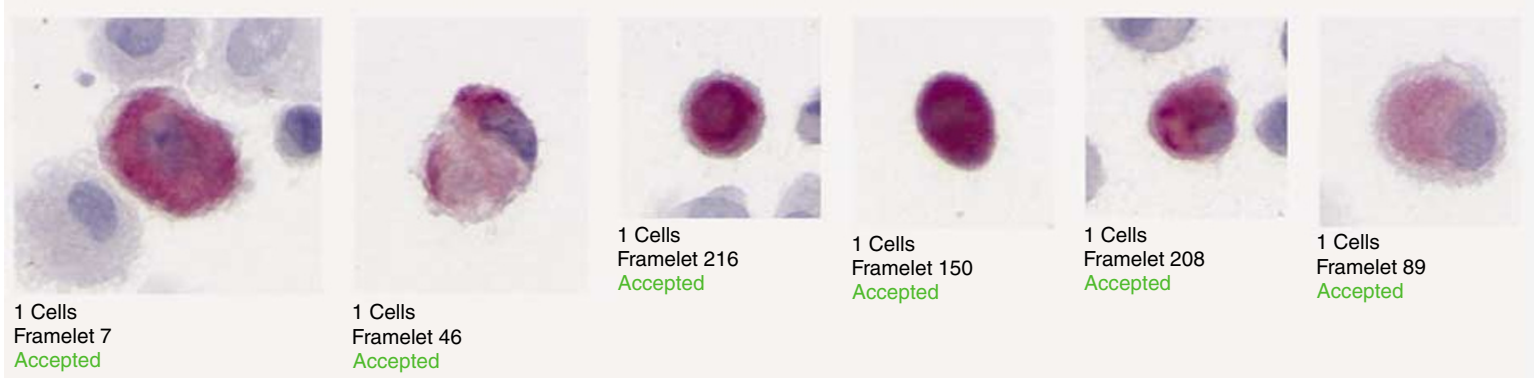

\section{Patient 490}
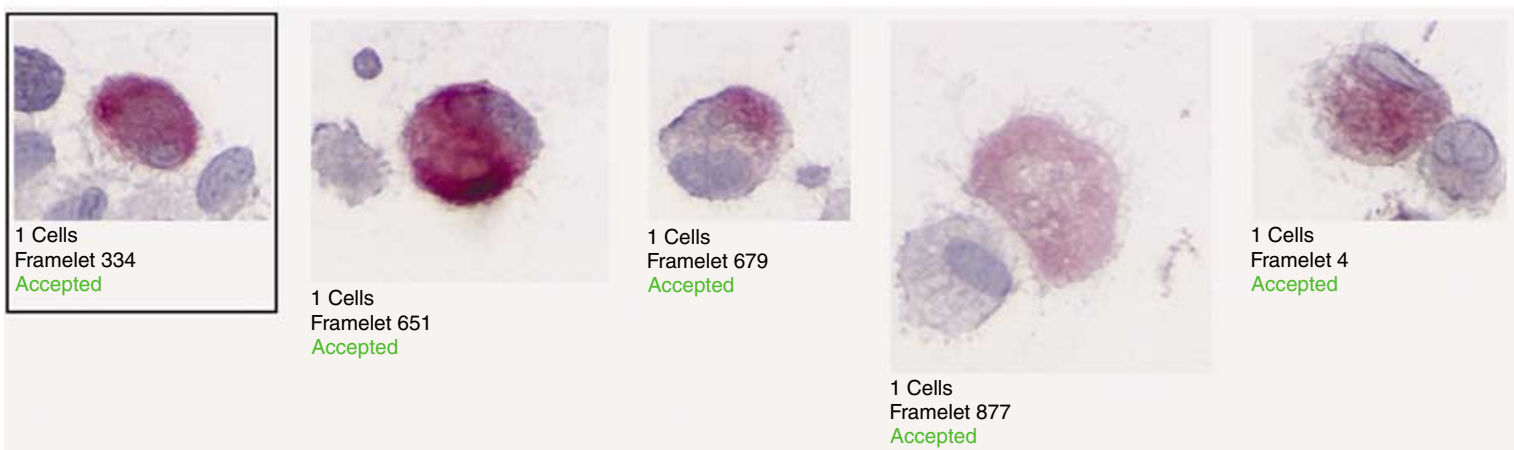

1 Cells Accepted

Accepted
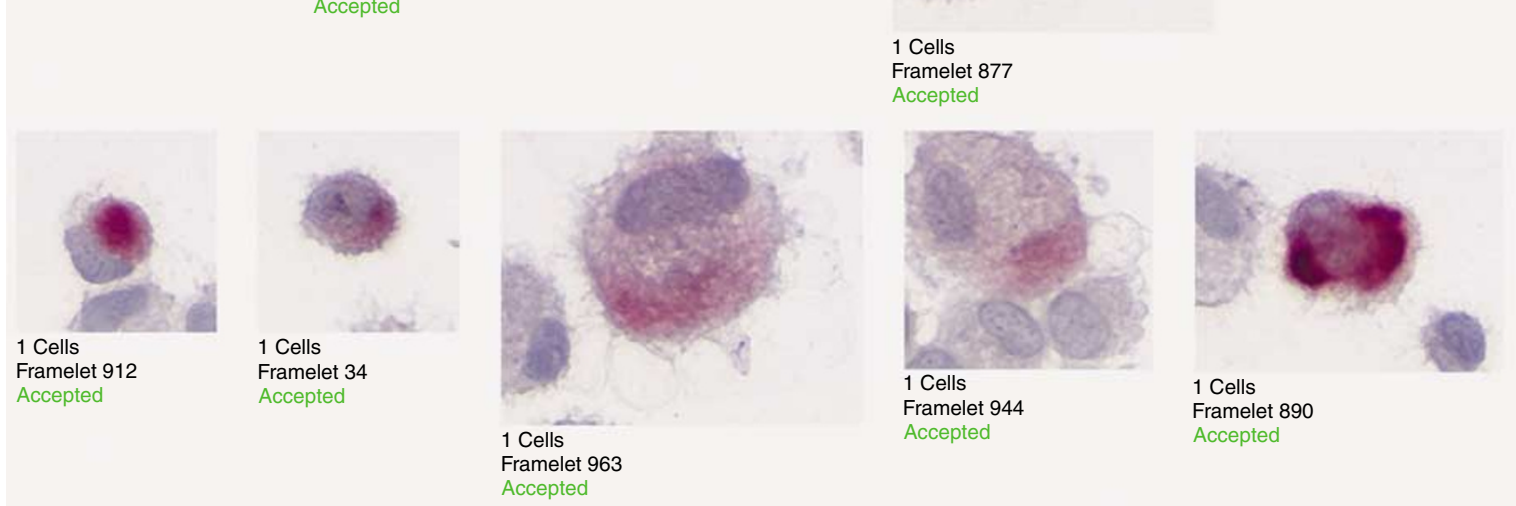

Figure I Example of automated detection and montage of CK-positive cells in vitro expanded bone marrow sample of two stage IV patients (patient's 344 and 490).

the CK-negative group was statistically significant on KaplanMeier analysis $(P=0.04)$ (Figure $3 \mathrm{~A})$.

\section{DISCUSSION}

The present study investigates the clinical relevance of the extent of in vitro expansion of CK-positive cells present in bone marrow aspirate, under adapted conditions published by Pantel et al (1995) and Solakoglu et al (2002). Compared to Solakoglu's study, our study included more metastatic patients and a smaller total number of patients. However, our series is homogeneous in terms of the type of cancer, as all patients presented breast cancer, in contrast with Solakoglu's study which included a very heterogeneous population including prostate, renal and breast cancers. The detection of isolated tumour cells in the bone marrow of patients with metastatic breast cancer has also been demonstrated to be a factor of poor prognosis (Janni et al, 2000).

Cultured bone marrow cells may nonspecifically express CK detected by anticytokeratin antibodies. Pantel et al (1995) using the same antibody as that used in our study excluded this possibility by using a negative control and confirmation of tumour 
Table 2 Comparison of detection of CK-positive cells at initial screening and detection of CK-positive cells grown in culture of bone marrow, in 29 primary patients (stage I-III), in 3 I metastatic patients (stage IV) and in the whole population $(N=60)$

\begin{tabular}{llccc}
\hline & $\begin{array}{l}\text { CK-positive cells } \\
\text { grown in culture }\end{array}$ & $\begin{array}{c}\text { Negative primary } \\
\text { screening }\end{array}$ & $\begin{array}{c}\text { Positive primary } \\
\text { screening }\end{array}$ & \\
\hline Primary & Negative & 8 & 4 & $12(41 \%)$ \\
$(N=29)$ & Positive & 10 & 7 & $17(59 \%)$ \\
& & $18(62 \%)$ & $11(38 \%)$ & $P=0.66$ \\
Metastatic & Negative & 4 & 4 & $8(26 \%)$ \\
$(N=31)$ & Positive & 4 & 19 & $23(74 \%)$ \\
& & $8(26 \%)$ & $23(74 \%)$ & $P=0.08$ \\
All patients & Negative & 12 & 8 & $20(33.3 \%)$ \\
$(N=60)$ & Positive & 14 & 26 & $40(66.6 \%)$ \\
& & $26(43.3)$ & $34(56.6)$ & $P=0.065$ \\
\hline
\end{tabular}

Table 3 Correlation between UICC stage of the patients and number of CK-positive cells detected on $3 \times 10^{6} \mathrm{MNCS}$ on initial screening. Lack of correlation between UICC stage of the patients and extent of in vitro expansion of CK-positive cells

\begin{tabular}{|c|c|c|c|c|}
\hline \multirow[b]{2}{*}{ Stage } & \multicolumn{2}{|c|}{ CK-positive primary screening } & \multicolumn{2}{|c|}{ CK-positive culture screening } \\
\hline & Number of cells/3 $\times 10^{6}$ & \pm s.d. & Number of cells $/ 10^{6}$ & \pm s.d. \\
\hline$\|(n=17)$ & 1.5 & 3.9 & 10.2 & 24.1 \\
\hline III $(n=12)$ & 2 & 5.7 & 11.2 & 17.6 \\
\hline IV $(n=31)$ & 105.8 & 247.7 & 31.2 & 89.1 \\
\hline Total $(n=60)$ & 55.5 & 84.3 & 21.3 & 66.0 \\
\hline Kruskal-Wallis & $P=0.0013$ & & $P=0.21$ & \\
\hline
\end{tabular}

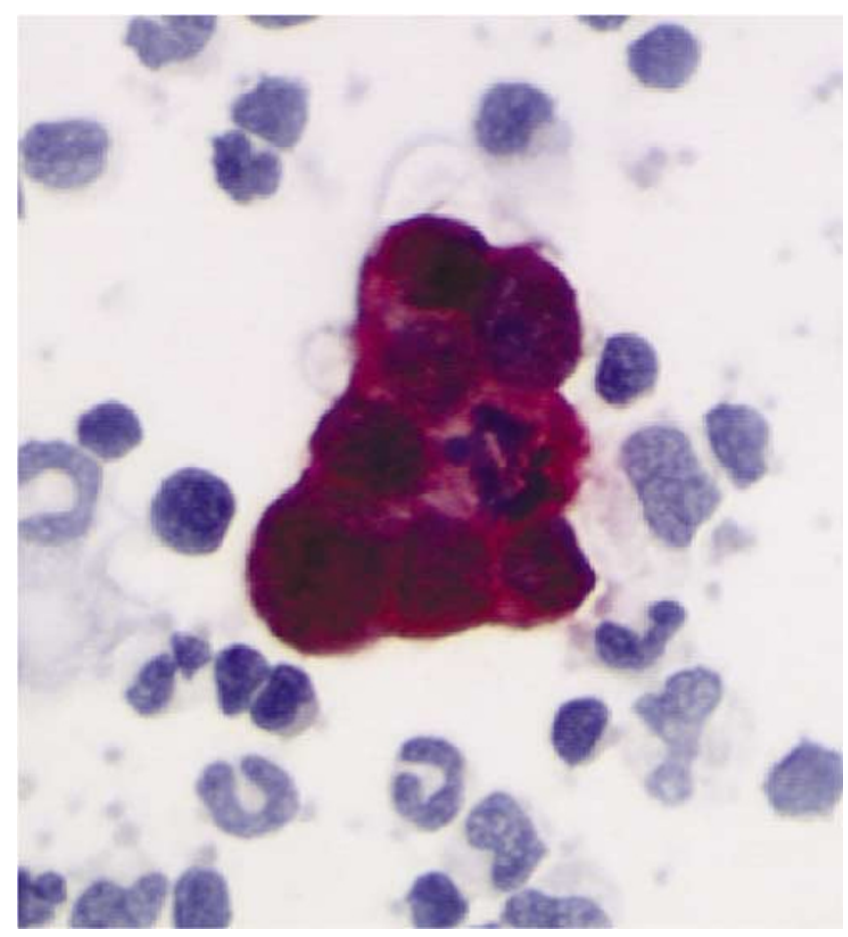

Figure 2 Cells in mitosis in a cluster detected in a stage IIB patient at primary screening.

status of stained cells by FISH. Nonspecific immunostaining was eliminated in our series by staining the same number of control slides under the same conditions with a nonrelevant primary antibody (anti-FITC) for each case.
The number of in vitro expanded CK-positive cells was not correlated with the number of CK-positive cells on bone marrow aspirates. Pantel et al (1995) demonstrated the variable growth kinetics of these tumours. The number of CK-positive cells on culture screening may therefore not be related to the various bone marrow loads observed among individual patients, but could reflect the in vitro proliferative potential of these cells. The concentration of tumour cells in culture can then increase as a result of proliferation of tumour cells in culture of nonadherent bone marrow cells. A significant number of bone marrow samples (14 patients, 23\%), negative on primary screening, became positive after cell culture. This could be explained by the number of bone marrow cells plated in culture flasks $\left(10\right.$ to $\left.30 \times 10^{6}\right)$ that is higher than the number of bone marrow cells examined on primary screening $\left(3 \times 10^{6}\right)$. These cultures were grown under almost limiting dilution conditions, that is, fewer than 10 tumour cells were plated per flask, indicating that micrometastatic cells can inherit a strong growth potential. Culture techniques could increase the sensitivity of detection of occult tumour cells in human bone marrow about 100-fold (Joshi et al, 1990). In a previous study published by Ross et al (1993), ICC detection of tumour involvement in bone marrow and peripheral blood stem cell collections (PBSC) was significant with in vitro clonogenic growth $(P<0.0001)$. The incidence and viability of tumour cell involvement in PBSC and bone marrow were studied in 48 patients with locally advanced or metastatic breast cancer enrolled on highdose chemotherapy programmes. In culture experiments, clonogenic tumour colonies grew in 21 out of 26 immunocytochemically positive specimens. No tumour colony growth was detected in 30 out of 32 immunochemically negative specimens. In contrast, in our study, several cases positive on primary screening were negative after culture expansion. This could indicate that the cells initially detected were unable to proliferate, but it could also be due to failure of trypsinisation, leaving the tumour cells adherent 
A

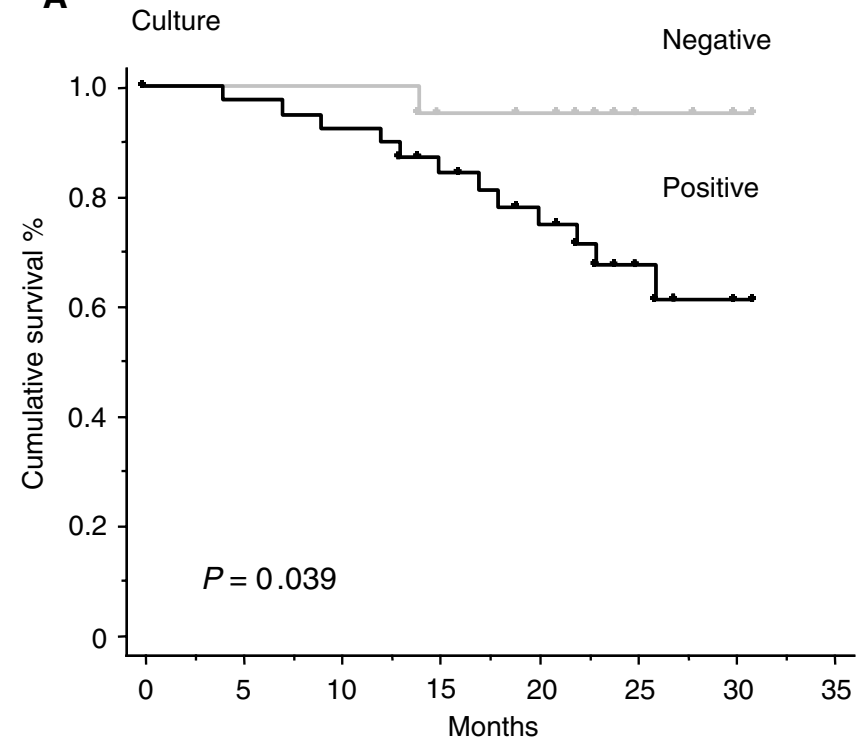

B

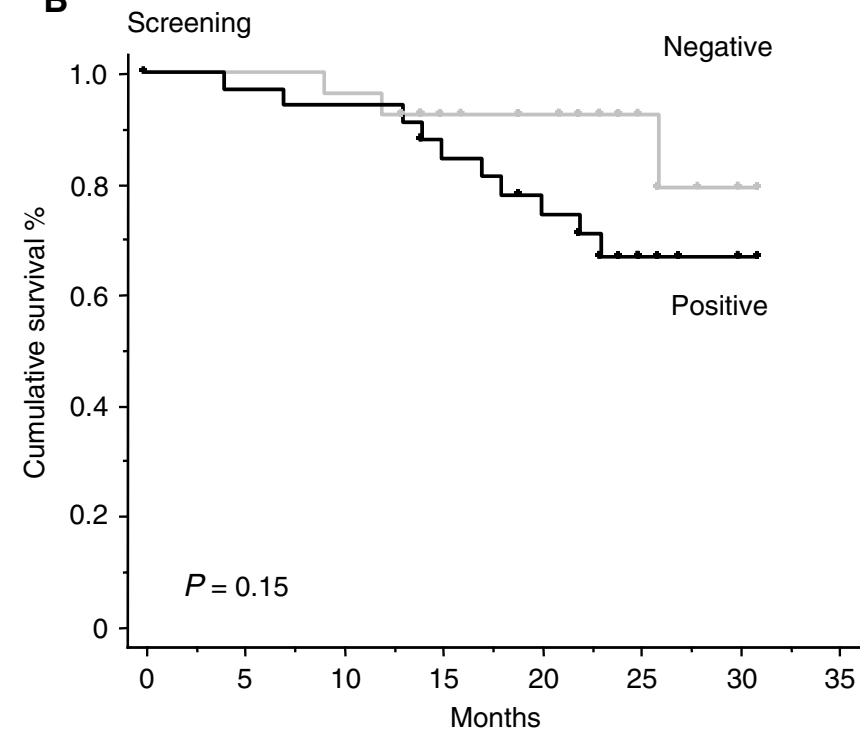

Figure 3 (A) Overall survival according to detection of CK-positive cells grown in culture of bone marrow (60 patients). (B) Overall survival according to the detection of CK-positive cells at initial screening of bone marrow sample (60 patients).

to the culture flask. Examination of the slides after staining may also not completely reflect the contents of the culture flask. In comparison with native tumour cells, cells shed from a tumour were less clonogenic, more apoptotic and less tumorigenic (Swartz et al, 1999). The degree of anchorage-independent growth of tumour cells can predict their biological behaviour and metastatic potential in vivo (Li et al, 1989; O'Sullivan et al, 1999).

We found a statistically significant relationship between the in vitro extent of expansion of micrometastatic tumour cells and patient outcome, essentially the survival of metastatic patients. We did not observe any difference in survival or disease-free survival because of the small number of nonmetastatic patients in this series and the short follow-up. All the patients who died during the follow-up were the metastatic patients. The cells that were expanded may be sourced from the secondary tumour rather than the primary. However, the extent of in vitro expansion of CKpositive cells was a better prognostic indicator than simple detection of these cells in bone marrow samples. Similar data have been obtained for the expression of HER2 and urokinase plasminogen activator receptor in bone marrow (Heiss et al., 1995; Allgayer et al, 1997; Braun et al, 2001). Bone marrow cells were cultured under specific conditions: cultures were performed under hypoxic conditions $\left(4 \% \mathrm{O}_{2}\right.$ instead of $\left.20 \% \mathrm{O}_{2}\right)$, as hypoxia stimulates carcinoma invasiveness by upregulating UPAR expression on the cell surface (Graham et al, 1999).

Analysis of cultured micrometastatic tumour cells could provide a greater number of CK-positive cells available for subsequent molecular analyses. Analysis of cultured CK-positive cells could contribute to the identification of the metastatic stem cells responsible for the formation of overt metastases. Cell lines have also been established from bone marrow micrometastases (Rye et al, 1996; Putz et al., 1999), but the culture process could lead to selection of those cell clones that adapt best to the culture conditions.

In all the large published series, not all patients with CK-positive cells in bone marrow develop clinical metastases. CK-positive cells appear to have a heterogeneous phenotype (Braun et al, 1999) and their genotype has also been shown to be heterogeneous (Klein et al, 2002). Klein et al reported a high genetic variability in minimal residual cancer, particularly in terms of chromosomal imbalances by using comparative genomic hybridisation. Two individual tumour cells from the same patient may not be identical, supporting the concept of genetic instability.

Solakoglu et al (2002) has suggested that larger prospective clinical trials be performed in order to confirm the clinical relevance of the in vitro extent of expansion of CK-positive cells. This type of trial would be very difficult to organise in view of the very specific culture conditions and the fairly long time required for detection of tumour cells in culture. However, a better understanding of the biology of micrometastatic cells is necessary to increase the precision of detection in bone marrow.

\section{ACKNOWLEDGEMENTS}

We thank Thierry Duchêne for technical assistance. This work was supported by the 'Programme Incitatif et Coopératif Micrométastases' of the Institut Curie.

\section{REFERENCES}

Allgayer H, Heiss MM, Riesenberg R, Grützner KU, Tarabichi A, Babic R, Schildberg FW (1997) Urokinase plasminogen activator receptor (uPA$\mathrm{R})$ : one potential characteristic of metastatic phenotypes in minimal residual tumor disease. Cancer Res 57: $1394-1399$

Bauer KD, de la Torre-Bueno J, Diel IJ, Hawes D, Decker WJ, Priddy C, Bossy B, Ludmann S, Yamamoto K, Masih AS, Espinoza FP, Harrington DS (2000) Reliable and sensitive analysis of occult bone marrow metastases using automated cellular imaging. Clin Cancer Res 6: $3552-3559$

Borgen E, Naume B, Nesland JM, Kvalheim G, Beiske K, Fodstad O, Diel I, Solomayer EF, Theocharous P, Coombes RC, Smith BM, Wunder E, Marolleau JP, Garcia J, Pantel K (1999) Standardisation of the immunocytochemical detection of cancer cells in BM and blood: I. Establishment of objective criteria for the evaluation of immunostained cells. The European ISHAGE Working Group for Standardization of Tumor Cell Detection. Cytotherapy 1: 377-388

Braun S, Hepp F, Sommer HL, Pantel K (1999) Tumorantigen heterogeneity of disseminated breast cancer cells: 
implications for immunotherapy of minimal residual disease. Int $J$ Cancer 84: $1-5$

Braun S, Kentenich C, Janni W, Hepp F, de Waal J, Willgeroth F, Sommer $\mathrm{H}$, Pantel K (2000a) Lack of effect of adjuvant chemotherapy on the elimination of single dormant tumor cells in bone marrow of high-risk breast cancer patients. J Clin Oncol 18: 80-86

Braun S, Muller M, Hepp F, Schlimok G, Riethmuller G, Pantel K (1998) Re: micrometastatic breast cancer cells in bone marrow at primary surgery: prognostic value in comparison with nodal status [letter; comment]. J Natl Cancer Inst 90: 1099-1101

Braun S, Pantel K (1999) Biological characteristics of micrometastatic cancer cells in bone marrow. Cancer Metastasis Rev 18: 75-90

Braun S, Pantel K, Muller P, Janni W, Hepp F, Kentenich CR, Gastroph S, Wischnik A, Dimpfl T, Kindermann G, Riethmuller G, Schlimok G (2000b) Cytokeratin-positive cells in the bone marrow and survival of patients with stage I, II, or III breast cancer. $N$ Engl J Med 342: 525-533

Braun S, Schlimok G, Heumos I, Schaller G, Riethdorf L, Riethmuller G, Pantel K (2001) ErbB2 overexpression on occult metastatic cells in bone marrow predicts poor clinical outcome of stage I-III breast cancer patients. Cancer Res 61: 1890-1895

Cordell JL, Falini B, Erber WN, Ghosh AK, Abdulaziz Z, MacDonald S, Pulford KA, Stein H, Mason DY (1984) Immunoenzymatic labeling of monoclonal antibodies using immune complexes of alkaline phosphatase and monoclonal anti-alkaline phosphatase (APAAP complexes). J Histochem Cytochem 32: 219-229

Diel IJ, Kaufmann M, Costa SD, Holle R, von Minckwitz G, Solomayer EF, Kaul S, Bastert G (1996) Micrometastatic breast cancer cells in bone marrow at primary surgery: prognostic value in comparison with nodal status. J Natl Cancer Inst 88: $1652-1658$

Funke I, Schraut W (1998) Meta-analyses of studies on bone marrow micrometastases: an independent prognostic impact remains to be substantiated. J Clin Oncol 16: $557-566$

Gebauer G, Fehm T, Merkle E, Beck EP, Lang N, Jager W (2001) Epithelial cells in bone marrow of breast cancer patients at time of primary surgery: clinical outcome during long-term follow-up. J Clin Oncol 19: $3669-3674$

Gerber B, Krause A, Muller H, Richter D, Reimer T, Makovitzky J, Herrnring C, Jeschke U, Kundt G, Friese K (2001) Simultaneous immunohistochemical detection of tumor cells in lymph nodes and bone marrow aspirates in breast cancer and its correlation with other prognostic factors. J Clin Oncol 19: 960-971

Graham CH, Forsdike J, Fitzgerald CJ, Macdonald-Goodfellow S (1999) Hypoxia-mediated stimulation of carcinoma cell invasiveness via upregulation of urokinase receptor expression. Int J Cancer 80: 617-623

Harbeck N, Untch M, Pache L (1994) Tumour cell detection in the bone marrow of breast cancer patients at primary therapy: results of a 3-year median follow-up. Br J Cancer 69: 566-571

Heiss MM, Allgayer H, Gruetzner U, Funke I, Babic R, Jauch KW, Schildberg FW (1995) Individual development and uPA-receptor expression of disseminated tumour cells in bone marrow: a reference to early systemic disease in solid cancer. Nature Med 1: 1035-1039

Janni W, Gastroph S, Hepp F, Kentenich C, Rjosk D, Schindlbeck C, Dimpfl T, Sommer H, Braun S (2000) Prognostic significance of an increased number of micrometastatic tumor cells in the bone marrow of patients with first recurrence of breast carcinoma. Cancer 88: 2252-2259

Joshi SS, Novak DJ, Messbarger L, Maitreyan V, Weisenburger DD, Sharp JG (1990) Levels of detection of tumor cells in human bone marrow with or without prior culture. Bone Marrow Transplant 6: 179-183
Kaplan EL, Meier P (1958) Nonparametric estimation from incomplete observations. J Am Stat Assoc 53: $457-481$

Klein CA, Blankenstein TJ, Schmidt-Kittler O, Petronio M, Polzer B, Stoecklein NH, Riethmuller G (2002) Genetic heterogeneity of single disseminated tumour cells in minimal residual cancer. Lancet 360: 683-689

Li L, Price JE, Fan D, Zhang RD, Bucana CD, Fidler IJ (1989) Correlation of growth capacity of human tumor cells in hard agarose with their in vivo proliferative capacity at specific metastatic sites. J Natl Cancer Inst 81: $1406-1412$

Mansi JL, Gogas H, Bliss JM, Gazet JC, Berger U, Coombes RC (1999) Outcome of primary-breast-cancer patients with micrometastases: a long-term follow-up study. Lancet 354: 197-202

O'Sullivan GC, Sheehan D, Clarke A, Stuart R, Kelly J, Kiely MD, Walsh T, Collins JK, Shanahan F (1999) Micrometastases in esophagogastric cancer: high detection rate in resected rib segments. Gastroenterology 116: $543-548$

Pantel K, Dickmanns A, Zippelius A, Klein C, Shi J, Hoechtlen Vollmar W, Schlimok G, Weckermann D, Oberneder R, Fanning E, Riethmüller G (1995) Establishment of micrometastatic carcinoma cell lines: a novel source of tumor cell vaccines. J Natl Cancer Inst 87: $1162-1168$

Pantel K, Schlimok G, Braun S, Kutter D, Lindemann F, Schaller G, Funke I, Izbicki JR, Riethmüller G (1993) Differential expression of proliferationassociated molecules in individual micrometastatic carcinoma cells. $J$ Natl Cancer Inst 85: 1419-1424

Putz E, Witter K, Offner S, Stosiek P, Zippelius A, Johnson J, Zahn R, Riethmuller G, Pantel K (1999) Phenotypic characteristics of cell lines derived from disseminated cancer cells in bone marrow of patients with solid epithelial tumors: establishment of working models for human micrometastases. Cancer Res 59: $241-248$

Ross AA, Cooper BW, Lazarus HM, Mackay W, Moss TJ, Ciobanu N, Tallman MS, Kennedy MJ, Davidson NE, Sweet D, Winter C, Akard L, Jansen J, Cepelan E, Meagher RC, Herzig RH, Klumpp TR, Kahn DG, Warner ME (1993) Detection and viability of tumor cells in peripheral blood stem cell collections from breast cancer patients using immunocytochemical and clonogenic assay techniques. Blood 82: 2605-2610

Rye PD, Norum L, Olsen DR, Garman-Vik S, Kaul S, Fodstad O (1996) Brain metastasis model in athymic nude mice using a novel MUC1secreting human breast-cancer cell line, MA11. Int J Cancer 68: 682-687

Schwartz G (1991) Cytomorphology and cell yield in a new centrifugal technique allowing the collection of the cell-free supernatant. Lab Med 15: $45-50$

Solakoglu O, Maierhofer C, Lahr G, Breit E, Scheunemann P, Heumos I, Pichlmeier U, Schlimok G, Oberneder R, Kollermann MW, Kollermann J, Speicher MR, Pantel K (2002) Heterogeneous proliferative potential of occult metastatic cells in bone marrow of patients with solid epithelial tumors. Proc Natl Acad Sci USA 99: 2246-2251

Stigbrand T, Andres C, Bellanger L, Bishr Omary M, Bodenmuller H, Bonfrer H, Brundell J, Einarsson R, Erlandsson A, Johansson A, Leca JF, Levi M, Meier T, Nap M, Nustad K, Seguin P, Sjodin A, Sundstrom B, van Dalen A, Wiebelhaus E, Wiklund B, Arlestig L, Hilgers J (1998) Epitope specificity of 30 monoclonal antibodies against cytokeratin antigens: the ISOBM TD5-1 Workshop. Tumour Biol 19: 132-152

Swartz MA, Kristensen CA, Melder RJ, Roberge S, Calautti E, Fukumura D, Jain RK (1999) Cells shed from tumours show reduced clonogenicity, resistance to apoptosis, and in vivo tumorigenicity. $\mathrm{Br} J$ Cancer 81: $756-759$ 